CIRJE-F-624

\title{
Generating a Target Payoff Distribution with the Cheapest Dynamic Portfolio: An Application to Hedge Fund Replication
}

\author{
Akihiko Takahashi \\ University of Tokyo \\ Kyo Yamamoto \\ GCI Asset Management, Inc.
}

June 2009; Revised in March 2010 and February 2013

CIRJE Discussion Papers can be downloaded without charge from:

http://www.cirje.e.u-tokyo.ac.jp/research/03research02dp.html

Discussion Papers are a series of manuscripts in their draft form. They are not intended for circulation or distribution except as indicated by the author. For that reason Discussion Papers may not be reproduced or distributed without the written consent of the author. 


\title{
Generating a Target Payoff Distribution with the Cheapest Dynamic Portfolio: An Application to Hedge Fund Replication*
}

\author{
Akihiko Takahashi†and Kyo Yamamoto
}

First draft: June 16, 2009

This version: February 17, 2013

\footnotetext{
*This research is supported by CARF (Center for Advanced Research in Finance) and the global COE program "The research and training center for new development in mathematics." We are very grateful to two anonymous referees for their precious comments, which have improved the previous version of this paper substantially. Also, we thank Hideki Yamauchi and Takahiko Suenaga at GCI Asset Management Inc. and Tetsuya Aoki at GCI Investment Management Singapore Pte. Ltd. for their constant support. All the contents expressed in this research are solely those of the authors and do not represent any views or opinions of any institutions. The authors are not responsible or liable in any manner for any losses and/or damages caused by the use of any contents in this research. Takahashi and Yamamoto (2010) is the summary of this research. It is based on the presentation entitled "A New Hedge Fund Replication Method with the dynamic Optimal Portfolio” at Global Conference on Business and Finance 2010, Kailua-Kona, Hawaii.

${ }^{\dagger}$ Graduate School of Economics, The University of Tokyo

${ }^{\ddagger}$ GCI Asset Management, Inc.
} 


\begin{abstract}
This paper provides a new method to construct a dynamic optimal portfolio for asset management. This method generates a target payoff distribution using the cheapest dynamic trading strategy. As a practical example, the method is applied to hedge fund replication. This dynamic portfolio strategy is regarded as an extension of a hedge fund replication methodology that was developed by Kat and Palaro (2005a, b) and Papageorgiou, Remillard and Hocquard (2008) to address multiple trading assets with both long and short positions. Empirical analyses show that such an extension significantly improves the performance of replication in practice.
\end{abstract}

\title{
1 Introduction
}

Modern portfolio theory has been applied to asset management in practice since the seminal works of Markowitz (1952) and Merton (1969, 1971). This paper considers a tractable portfolio construction problem for portfolio managers. While Markowitz (1952) proposed a portfolio optimization method where the portfolio that minimizes its variance under a given expected return is chosen, this paper presents a method to create the dynamic portfolio that attains a target payoff distribution with the minimum cost in a continuoustime complete market. Moreover, this cost minimization problem is shown to be equivalent to a standard expected utility maximization problem that was studied by Merton $(1969,1971)$.

Practical performance evaluations of mutual and hedge funds are usually based upon the basic statistics of their returns, such as certain orders of moments and their combinations (e.g., Sharpe ratio). In particular, many researchers have pointed out that hedge fund returns exhibit non-normality (see, for example, Malkiel and Saha (2005) and Hakamada et al. (2007)). Thus, high orders of moments should be also considered for investment in hedge funds (see, for example, Gregoriou and Kaiser (2006) and Cvitanić, Polimenis and Zapatero (2008)). Dybvig (1988a) proposed a distributional analysis approach to evaluating investment performances. This approach modified the Sharpe and Jensen measures by incorporating all of the moments of a return distribution.

Our method extends the distributional analysis approach initiated by Dybvig (1988a). Theorem 1 in Dybvig (1988a) claims that the cheapest 
way to attain a given payoff is to allocate terminal wealth in the reverse order of the state price density in an equally probable finite state complete market. This article extends that claim to a continuous-time complete market framework. The uniqueness of the cheapest payoff is also proven. Theorem 2 in Dybvig (1988a) asserts that a trading strategy maximizes an increasing and concave von Neumann-Morgenstern utility in an equally probable finite state complete market if and only if it is the cheapest strategy among those that generate the same payoff distribution. This paper shows a similar result holds in a continuous-time complete market setting, as well. Hence, it is ensured that our portfolio optimization problem is equivalent to an expected utility maximization problem.

Dybvig (1988a) intended an extension of the capital asset pricing model (CAPM) and techniques for evaluating investment performance and testing its efficiency. In addition, Dybvig (1988b) used the distributional analysis to investigate the efficiency of the standard dynamic trading strategies that are followed by practitioners. However, this paper applies the idea to creating the dynamic optimal portfolio that achieves a target payoff distribution. For a target distribution, there is an interesting example: hedge fund replication.

Hedge fund replication products appeared in financial markets after the late 2000s. Large investment banks and asset management companies launched such products one after another. They have been developing their own original methods by employing highly sophisticated financial models and statistical methods. Some have developed replication techniques by collaborating with the pioneers in hedge fund research (see, for example, Takahashi and Yamamoto (2009) or Tuchschmid et al. (2010, 2012)). These products overcome some of the disadvantages of hedge fund investment: high cost, low transparency, and low liquidity. The importance of transparency and liquidity has been recognized since the subprime and Lehman shocks. Therefore, these products have gained increased attention. Tuchschmid et al. (2010, 2012) examined the performances of existing hedge fund clone products.

Many financial firms offer the clones of various investment strategies. Most of the replication products attempt to replicate the performances of aggregate composite hedge fund indexes from HFR, Dow Jones Credit Suisse, and so on. Others attempt to replicate the performances of some specific alternative investment strategies, such as equity long/short or market-neutral.

The existing methodologies for hedge fund replication can be categorized into the following three approaches: the rule-based, factor-based, and distribution-replicating approach. The rule-based approach mimics the typi- 
cal trading strategies that are employed by hedge funds. The trading rules in Duarte et al. (2007) can be regarded as rule-based fixed-income hedge fund clone techniques.

Factor-based cloning attempts to replicate the risk exposures of the target fund. If this method succeeds, then the return of the clone tracks that of the target fund on a month-to-month basis. Lo and Hasanhodzic (2007) and Fung and Hsieh (2007a, 2007b) studied this approach. Factor analysis techniques that have been developed for hedge funds since the late 1990s, such as that of Fung and Hsieh (1997, 2000, 2001) and Agarwal and Naik (2004), are directly applied to the replication.

The distribution-replicating approach aims to replicate the distributions of hedge fund returns. Amin and Kat (2003) first attempted this replication. However, hedge funds attract investors not only by their return distributions but also by their low correlations with the returns of traditional asset classes, such as stocks and bonds. Therefore, Kat and Palaro (2005a, b) extended the method to replicate both the distribution and the dependence structure on the investor's existing portfolio. Kat and Palaro (2005a, b) tried to replicate them through a dynamic trading of the investor's existing portfolio (approximated by a portfolio of stock index futures and bond futures) and another asset (replicating instrument). Papageorgiou et al. (2008) proposed an alternative way to perform Kat-Palaro's replication methodology by utilizing a hedging scheme for options in an incomplete market. The payoff distribution model can be applied to not only hedge fund replication but also to other investment strategies and risk managements. For example, Hocquard et al. (2012) applied it to portfolio insurance.

This article extends the work of Kat and Palaro (2005a, b) and Papageorgiou et al. (2008). While the methods developed by the two research papers use one replicating instrument with long positions only, our method allows multiple replicating instruments with both long and short positions for creating a replicating portfolio. With regard to practical applications, this paper replicates the return distributions of three different hedge fund strategies: managed futures, multi-strategy, and global macro indices from Dow Jones Credit Suisse. Such fund managers are seeking attractive investment opportunities in financial markets around the world. They employ dynamic trading strategies, including leverage and short sales, to make profits from such opportunities. Our methodology is able to reflect their investment behaviors. Managed futures funds gained high returns by taking short positions on risky assets in the months immediately following the bankruptcy 
of Lehman Brothers. As this new replication method allows for short positions, it is much easier to replicate a managed futures fund that shows good performance even in substantial bear markets. Historical out-of-sample simulations show that our scheme provides much better performances than do the replications with one replicating instrument and its long positions only.

This paper is organized as follows. The following section briefly reviews the existing distribution replication methodology with one replicating instrument and its long positions only. Section 3 describes the new dynamic portfolio optimization method, which is an extension of the distribution replication method to multiple replicating instruments with both long and short positions. Then, Section 4 applies the new method to the replication of hedge fund indexes. Section 5 concludes this paper. Finally, the Appendix gives the proofs of the theorems as well as the derivation of the dynamic replicating portfolios.

\section{Review of the Existing Distribution Repli- cation Methodology}

Before describing the new dynamic portfolio optimization method, let us review the distribution replication methodology developed by Kat and Palaro (2005a, b) and Papageorgiou et al. (2008), which helps us to understand the motivation of this study. Consider an investor who has been investing in traditional assets, such as stocks and bonds, and who plans to invest in a hedge fund. Assume that he/she is attracted to the hedge fund because of its return distribution and the structure of dependence upon his/her portfolio. Kat and Palaro (2005a, b) proposed to replicate the return distribution of the hedge fund and its dependence structure on the investor's existing portfolio by a dynamic trading strategy of the investor's portfolio (approximated by a portfolio of stock index futures and bond futures) and another asset (replicating instrument).

Let $S^{0}$ be a risk-free asset, $S^{1}$ be the investor's existing portfolio, and $S^{2}$ be a replicating instrument. Assume that $S^{1}$ is also tradable. Let 0 and $T$ be the start and terminal dates of the investment, respectively (for example, they are assumed to be the start and end of the month, respectively). Although Papageorgiou et al. (2008) assumed an incomplete market, this paper assumes a complete market. Suppose that the financial assets follow 
stochastic differential equations (SDEs)

$$
\begin{aligned}
d S_{t}^{0} & =r_{t} S_{t}^{0} d t \\
d S_{t}^{1} & =\mu_{t}^{1} S_{t}^{1} d t+\sigma_{t}^{11} S_{t}^{1} d W_{t}^{1}, \\
d S_{t}^{2} & =\mu_{t}^{2} S_{t}^{2} d t+\sigma_{t}^{21} S_{t}^{2} d W_{t}^{1}+\sigma_{t}^{22} S_{t}^{2} d W_{t}^{2},
\end{aligned}
$$

where $W_{t}^{1}$ and $W_{t}^{2}$ are independent Wiener processes, and $r_{t}, \mu_{t}^{i}$, and $\sigma_{t}^{i j}$ $(i, j=1,2)$ satisfy some measurability and integrability conditions. Normalize the initial asset values so that $S_{0}^{0}=S_{0}^{1}=S_{0}^{2}=1$. Then, the log returns of $S^{1}$ and $S^{2}$ are represented as $R_{T}^{1}=\log S_{T}^{1}$ and $R_{T}^{2}=\log S_{T}^{2}$, respectively.

Let $R_{T}$ be the random variable that represents the log return of the target hedge fund. Kat and Palaro $(2005 \mathrm{a}, \mathrm{b})$ proposed to replicate the joint distribution of $\left(R_{T}^{1}, R_{T}\right)$ through the dynamic trading of $S^{1}$ and $S^{2}$. They created a payoff function of $S^{1}$ and $S^{2}$ that has the same joint distribution as the target hedge fund, and they then replicated the payoff using a dynamic trading strategy for $S^{1}$ and $S^{2}$. They found function $\tilde{g}$, which satisfies the following equation.

$$
\mathbb{P}\left(R_{T}^{1} \leq a, \tilde{g}\left(R_{T}^{1}, R_{T}^{2}\right) \leq b\right)=\mathbb{P}\left(R_{T}^{1} \leq a, R_{T} \leq b\right) \text { for any } a, b,
$$

or equivalently,

$$
\mathbb{P}\left(\tilde{g}\left(R_{T}^{1}, R_{T}^{2}\right) \leq b \mid R_{T}^{1}=a\right)=\mathbb{P}\left(R_{T} \leq b \mid R_{T}^{1}=a\right) \text { for any } a, b .
$$

The function $\tilde{g}(\cdot, \cdot)$ is given by

$$
\tilde{g}(a, b)=F_{R_{T} \mid a}^{-1}\left(F_{R_{T}^{2} \mid a}(b)\right),
$$

where $F_{R_{T} \mid a}$ and $F_{R_{T}^{2} \mid a}$ are the conditional distribution functions of $R_{T}$ and $R_{T}^{2}$ under $R_{T}^{1}=a$. As the function of the asset prices, the payoff is represented as

$$
\hat{g}\left(S_{T}^{1}, S_{T}^{2}\right)=\exp \left\{\tilde{g}\left(\log S_{T}^{1}, \log S_{T}^{2}\right)\right\} .
$$

Payoff $\hat{g}\left(S_{T}^{1}, S_{T}^{2}\right)$ has the same joint distribution with the investor's portfolio as does the target hedge fund.

Once one has obtained the payoff function, then the replicating strategy encounters the same problem with pricing and hedging derivatives. The dynamic replicating strategy is given by the delta-hedging strategy of the payoff $\hat{g}\left(S_{T}^{1}, S_{T}^{2}\right)$. If the initial cost for the trading strategy is less (more) 
than one, then the target payoff distribution is realized by a lower (higher) cost. The remaining (shortage of) money is invested (funded) in the risk-free asset. This means that the shape of the probability density function can be replicated, but the mean return is higher (lower) than the target fund by the difference of the initial cost. In this case, the replicating instrument does (does not) include a greater investment opportunity than does the target hedge fund. Note that the payoff function $\hat{g}(\cdot, \cdot)$ is increasing with respect to the second argument. The delta-hedging strategy therefore never takes a short position for $S^{2}$. In pp. 17-18 of Kat and Palaro (2005a), the authors claim that users of this method should choose the replicating instrument $S^{2}$, which includes a positive expected return factor that is uncorrelated to the return of the investor's portfolio. The long positions for $S^{2}$ are thus justified. Therefore, the choice of a replicating instrument is a very important problem.

As just described, this methodology can replicate the shape of the probability density function, but it cannot fit the mean. If you found a greater investment opportunity than the target fund, the mean return would be superior, and vice versa. Therefore, the usage of only one asset is restrictive. Papageorgiou et al. (2008) synthesized multiple assets to create one replicating instrument by equal-weighing, but such a portfolio would not always be optimal. The extension of the investment universe would bring in higher mean returns. The dynamic portfolio strategy presented in the next section can be considered to be an extension of this distribution replication method to multiple replicating instruments with both long and short positions.

\section{The Cheapest Dynamic Portfolio that Gen- erates a Target Payoff Distribution}

Let us begin with a complete probability space $(\Omega, \mathcal{F}, \mathbb{P})$ with $n$-dimensional standard Brownian motion $W_{t}=\left(W_{t}^{1}, \cdots, W_{t}^{n}\right)^{\prime}, 0 \leq t \leq T$, where ' represents transposition so that $W_{t}$ is a column vector. $W_{0}=0$ is almost surely satisfied. Let $\left\{\mathcal{F}_{t}^{W}\right\}_{0 \leq t \leq T}$ be the filtration generated by $W_{t}$. This paper uses the augmented filtration

$$
\mathcal{F}_{t}=\sigma\left(\mathcal{F}_{t}^{W} \cup \mathcal{N}\right) \text { for any } t<T,
$$

where $\mathcal{N}$ denotes $\mathbb{P}$-null subsets of $\mathcal{F}_{T}^{W}$.

Let $S^{0}$ be a risk-free asset, $S^{1}$ be the investor's existing portfolio, and $S^{2}, \cdots, S^{n}$ be risky assets. Suppose that the price processes of the financial 
assets $S^{i}(i=0, \cdots, n),\left\{S_{t}^{i}\right\}_{t=0}^{T}$, follow SDEs

$$
\begin{gathered}
d S_{t}^{0}=r_{t} S_{t}^{0} d t \\
d S_{t}^{i}=\mu_{t}^{i} S_{t}^{i} d t+\sum_{j=1}^{i} \sigma_{t}^{i j} S_{t}^{i} d W_{t}^{j} \quad(i=1, \cdots, n),
\end{gathered}
$$

where $r_{t}, \mu_{t}^{i}$, and $\sigma_{t}^{i j}$ are progressively measurable and satisfy

$$
\begin{aligned}
& \int_{0}^{T}\left|r_{t}\right| d t<\infty \text { a.s. } \\
& \int_{0}^{T}\left|\mu_{t}^{i}\right| d t<\infty \text { a.s. } \\
& \int_{0}^{T}\left(\sigma_{t}^{i j}\right)^{2} d t<\infty \text { a.s. }
\end{aligned}
$$

for any $1 \leq j \leq i \leq n$.

All of the initial asset values are normalized, so that $S_{0}^{0}=\cdots=S_{0}^{n}=1$. The following notations for $n$-dimensional vectors and an $n \times n$ matrix are introduced. $S_{t}=\left(S_{t}^{1}, \cdots, S_{t}^{n}\right)^{\prime}, \mu_{t}=\left(\mu_{t}^{1}, \cdots, \mu_{t}^{n}\right)^{\prime}, \overrightarrow{1}=(1, \cdots, 1)^{\prime}$, and

$$
\sigma_{t}=\left(\begin{array}{ccc}
\sigma_{t}^{11} & & O \\
\vdots & \ddots & \\
\sigma_{t}^{n 1} & \cdots & \sigma_{t}^{n n}
\end{array}\right)
$$

where' represents transposition so that $S_{t}, \mu_{t}$ and $\overrightarrow{1}$ are column vectors.

Suppose that $\sigma_{t}$ is almost surely invertible. There then exists the unique market price of the risk

$$
\theta_{t}=\sigma_{t}^{-1}\left(\mu_{t}-r_{t} \overrightarrow{1}\right)
$$

In other words, the financial market is complete. The financial market is denoted by $\mathcal{M}=(r, \mu, \sigma)$.

In a complete market $\mathcal{M}$, the unique state price density process is given by

$$
H_{t}=\exp \left\{-\int_{0}^{t} r_{u} d u-\frac{1}{2} \int_{0}^{t}\left\|\theta_{u}\right\|^{2} d u-\int_{0}^{t} \theta_{u}^{\prime} d W_{u}\right\} .
$$


The no-arbitrage price of any $\mathcal{F}_{T}$-measurable payoff $X$ is given by $x=$ $E\left[H_{T} X\right] . X$ can be replicated by a dynamic trading of the financial assets with initial cost $x$ (see, for example, Karatzas and Shreve (1998)).

When multiple risk assets are tradable, then there are infinitely many payoffs that have a target distribution. This paper proposes to choose the cheapest of these payoffs. Dybvig (1988a) showed that the cheapest way to attain a given payoff is to allocate the terminal wealth in the reverse order of the state price density in an equally probable finite state complete market. As will be seen later, this claim is also valid in our setting under some conditions. In the following, we find the cheapest payoffs that attain a target marginal distribution and a joint distribution with the investor's existing portfolio.

First, let us find the cheapest payoff that has a target marginal distribution. Let $\xi$ be a random payoff that has a target distribution. For convenience, the minus logarithm state price density process $L_{t}$ is introduced as follows.

$$
L_{t}=-\log H_{t}=\int_{0}^{t} r_{u} d u+\frac{1}{2} \int_{0}^{t}\left\|\theta_{u}\right\|^{2} d u+\int_{0}^{t} \theta_{u}^{\prime} d W_{u} .
$$

Because Arrow-Debreu securities are tradable in a complete market, this paper proposes to create a payoff using $H_{T}$ (or $L_{T}$ ). Let $F_{\xi}$ and $F_{L_{T}}$ denote the distribution functions of $\xi$ and $L_{T}$, respectively. Assume that $F_{\xi}$ is an invertible function. If $X$ is defined as follows, then $X$ has the same distribution as $\xi$.

$$
X=f\left(L_{T}\right)
$$

where

$$
f(l)=F_{\xi}^{-1}\left(F_{L_{T}}(l)\right) .
$$

Because $F_{\xi}^{-1}$ and $F_{L_{T}}$ are increasing, the payoff $X$ increases in $L_{T}$, and therefore it is in the reverse order of the state price density $H_{T}$. Thus, $X$ is the cheapest payoff among the payoffs that have the same distribution as $\xi$. The next theorem asserts that $X$ is the unique, cheapest payoff among the random variables that has the same distribution as $\xi$.

Theorem 1 Assume that $\xi$ is a positive $\mathcal{F}_{T}$-measurable random variable, and $F_{\xi}$ and $F_{L_{T}}$ are continuous and strictly increasing. In a complete market $\mathcal{M}$, the unique cheapest payoff $X$ that has the same distribution as $\xi$ is given by equation (5). 
Proof. See Appendix 6.1.

Additionally, the choice of the cheapest payoff for some marginal distribution can be justified in terms of the expected utility maximization theory. It can be shown that the cost minimization for a marginal payoff distribution is equivalent to a von Neumann-Morgenstern utility maximization. Dybvig (1988a) proved this claim in the equally probable finite state setting. The next theorem proves this claim in a continuous-time framework.

Theorem 2 Assume that $\xi$ is a positive $\mathcal{F}_{T}$-measurable random variable, and $F_{\xi}$ and $F_{L_{T}}$ are continuous and strictly increasing. If $X$ is the cheapest payoff that has the same distribution with $\xi$ (i.e., $X$ is defined by equation (5)), then in a complete market $\mathcal{M}$, there exists a strictly increasing and strictly concave von Neumann-Morgenstern utility function $u(\cdot)$, such that (a) $\lim _{z \rightarrow+0} u^{\prime}(z)=+\infty$, (b) $\lim _{z \rightarrow+\infty} u^{\prime}(z)=0$, and the dynamic trading strategy that attains payoff $X$ is the optimal investment strategy for $u(\cdot)$.

Conversely, if a dynamic trading strategy maximizes a strictly increasing and strictly concave von Neumann-Morgenstern utility function $u(\cdot)$ that satisfies conditions (a) and (b), then it attains the cheapest payoff for some distribution.

Proof. See Appendix 6.2.

Next, let us find the cheapest payoff that has a target joint distribution with the investor's existing portfolio, $S^{1}$. Denote the conditional distribution functions of $\xi$ and $L_{T}$ under condition $S_{T}^{1}=s$ by $F_{\xi \mid s}$ and $F_{L_{T} \mid s}$, respectively. Assume that $F_{\xi \mid s}$ is invertible for any $s$. If $X$ is defined as follows, then $\left(S_{T}^{1}, X\right)$ has the same joint distribution as $\left(S_{T}^{1}, \xi\right)$.

$$
X=g\left(S_{T}^{1}, L_{T}\right)
$$

where

$$
g(s, l)=F_{\xi \mid s}^{-1}\left(F_{L_{T} \mid s}(l)\right) .
$$

Because $F_{\xi \mid s}^{-1}$ and $F_{L_{T} \mid s}$ are increasing, the payoff $X$ increases in $L_{T}$, and it is therefore in the reverse order of the state price density, $H_{T}$. Thus, $X$ is the cheapest payoff among the payoffs that have the same joint distribution with the investor's existing portfolio as $\xi$. The next theorem asserts that $X$ is the unique, cheapest payoff among the random variables whose joint distributions with $S_{T}^{1}$ are the same as $\xi$. 
Theorem 3 Assume that $\xi$ is a positive $\mathcal{F}_{T}$-measurable random variable, and $F_{\xi \mid s}$ and $F_{L_{T} \mid s}$ are continuous and strictly increasing for any $s>0$. In a complete market $\mathcal{M}$, the unique cheapest payoff $X$ among the random variables whose joint distributions with $S_{T}^{1}$ are the same as $\xi$ is given by equation (6).

Proof. See Appendix 6.3.

Let us derive the dynamic portfolio that replicates the cheapest payoffs. Let $\pi_{t}^{i}(i=0, \cdots, n)$ represent the money amount that is invested in asset $i$ at time $t$. The $n$-dimensional vector $\pi_{t}$ is defined by $\pi_{t}=\left(\pi_{t}^{1}, \cdots, \pi_{t}^{n}\right)^{\prime}$, which denotes a portfolio of risky assets. Let $x$ be the initial cost that is required to realize the cheapest payoff $X_{T}$ for some payoff distribution. The initial cost $x$ is invested in the financial assets by a dynamic self-financing trading strategy to generate the payoff $X_{T}$. In other words, the portfolio value at time $t, X_{t}$, satisfies

$$
X_{t}=\pi_{t}^{0}+\pi_{t}^{\prime} \overrightarrow{1}
$$

for any $t$. In a differential form, this is expressed as

$$
d X_{t}=r_{t} X_{t} d t+\pi_{t}^{\prime}\left(\mu_{t}-r_{t} \overrightarrow{1}\right) d t+\pi_{t}^{\prime} \sigma_{t} d W_{t} .
$$

The dynamic portfolio can be obtained concretely for the case of Markovian coefficients. See Appendix 6.4 for details. The following discussion in this paper applies a special case of Appendix 6.4, where $r, \mu$, and $\sigma$ are deterministic functions of the time $t$. The next proposition provides the dynamic replicating portfolios in this case.

Proposition 1 Assume that $r, \mu$ and $\sigma$ are deterministic functions of the time $t$. Then, in a complete market $\mathcal{M}$, the dynamic portfolio that generates payoff $f\left(L_{T}\right)$ is given by

$$
\pi_{t}^{M}=\sigma^{\prime}(t)^{-1} \phi_{t}^{M}
$$

where

$$
\phi_{t}^{M}=\frac{\theta(t)}{H_{t}} E_{t}\left[H_{T} f^{\prime}\left(L_{T}\right)\right] .
$$

The portfolio that attains the payoff $g\left(S_{T}^{1}, L_{T}\right)$ is given by

$$
\pi_{t}^{J}=\sigma^{\prime}(t)^{-1} \phi_{t}^{J}
$$


where $\phi_{t}^{J}=\left(\phi_{t}^{J 1}, \cdots, \phi_{t}^{J n}\right)$ is given by

$$
\begin{gathered}
\phi_{t}^{J 1}=\frac{\theta^{1}(t)}{H_{t}} E_{t}\left[H_{T} g_{2}\left(S_{T}^{1}, L_{T}\right)\right]+\frac{\sigma^{11}(t)}{H_{t}} E_{t}\left[H_{T} g_{1}\left(S_{T}^{1}, L_{T}\right) S_{T}^{1}\right], \\
\phi_{t}^{J i}=\frac{\theta^{i}(t)}{H_{t}} E_{t}\left[H_{T} g_{2}\left(S_{T}^{1}, L_{T}\right)\right], \text { for } i=2, \cdots, n,
\end{gathered}
$$

where $g_{i}(i=1,2)$ represents the partial derivative of $g$ with respect to the $i$-th argument.

Portfolios $\pi_{t}^{M}$ and $\pi_{t}^{J}$ are obtained if the conditional expectations in equations (8), (10), and (11) are evaluated.

The interpretations for the optimal portfolio constituent factors are as follows. As for $\pi_{t}^{M}, \frac{1}{H_{t}} E_{t}\left[H_{T} f^{\prime}\left(L_{T}\right)\right]$ is the present value of the sensitivity of the terminal payoff with respect to the change in $L_{T}$. This quantity corresponds to delta in the option theory. The size of the risky asset portfolio increases in this quantity. This factor contributes to generating the target distribution. In addition, the replicating strategy allocates the wealth to tradable assets according to the market price of the risk, $\theta^{i}(t)$. Through this process, the cheapest strategy is realized. The difference between $\pi_{t}^{J}$ and $\pi_{t}^{M}$ is the second term in equation (10). This is the present value of the sensitivity of the terminal payoff with respect to the change of $W_{T}^{1}$. This term contributes to the generation of the structure of dependence upon the investor's existing portfolio.

For the case of deterministic coefficients, the computational burden to obtain the dynamic replicating portfolio does not increase in its number of replicating instruments. To get the dynamic portfolio for the marginal distribution, the conditional expectation in equation (8) needs to be evaluated. All that we then need is the distribution of $L_{T}$ under the information given at times 0 and $t$. As $L_{T}$ follows a one-dimensional Gaussian distribution, the conditional expectation can be numerically computed by Monte Carlo simulations or one-dimensional numerical integration. To obtain the dynamic portfolio for the joint distribution, we need the conditional expectations of equations (10) and (11). They can be numerically computed by the Monte Carlo simulations or by two-dimensional numerical integrations because all that we need are the joint distributions of $\left(\log S_{T}^{1}, L_{T}\right)$ under the information given at times 0 and $t$. As $L_{t}$ is given by equation (4), $L_{T}$ is represented as

$$
L_{T}=\int_{0}^{T} r(t) d t+\frac{1}{2} \int_{0}^{T}\|\theta(t)\|^{2} d t+\int_{0}^{T} \theta^{1}(t) d W_{t}^{1}+\sum_{i=2}^{n} \int_{0}^{T} \theta^{i}(t) d W_{t}^{i}
$$


The distribution of $\sum_{i=2}^{n} \int_{0}^{T} \theta^{i}(t) d W_{t}^{i}$ under the information given at time 0 or $t$ is the Gaussian distribution with mean 0 or $\sum_{i=2}^{n} \int_{0}^{t} \theta^{i}(u) d W_{u}^{i}$ and variance $\sum_{i=2}^{n} \int_{0}^{T}\left\{\theta^{i}(u)\right\}^{2} d u$ or $\sum_{i=2}^{n} \int_{t}^{T}\left\{\theta^{i}(u)\right\}^{2} d u$, respectively. Therefore, the joint distributions of $\left(\log S_{T}^{1}, L_{T}\right)$ can be described by two-dimensional Gaussian distributions. Thus, the extension to multiple replicating instruments does not bring any computational disadvantage to the dynamic replicating portfolios.

\section{Application to Hedge Fund Replication}

This section applies the dynamic portfolio strategy developed in the previous section to the replication of hedge fund return distributions. In the framework of hedge fund replication, this is considered to be an extension of the hedge fund replication methodology developed Kat and Palaro (2005a, b) and Papageorgiou et al. (2008) to multiple replicating instruments with both long and short positions. Empirical studies are performed in the following subsections.

First, the data used in this study are described. Then, the strategy descriptions of three hedge fund strategies that are replicated in this study are given. Next, the empirical methodologies are explained. Finally, out-ofsample replicating simulations for the three hedge fund strategies are performed.

\subsection{Data Description}

This paper uses Dow Jones Credit Suisse Hedge Fund Indices as representative measurements of hedge fund performances. The monthly return data can be downloaded from its homepage. Table 4 exhibits the summary statistics on the monthly log returns of Dow Jones Credit Suisse Hedge Fund Indices. The table shows the statistics calculated using three different data periods: (a) the total period (1995-2011), (b) the first period (1995-2000), and (c) the second period (2001-2011). By comparing the moments of the return distributions in the different periods in Table 4, most of the hedge fund strategies have historically changed their return distributions.

In practice, the return distribution of a target hedge fund is estimated by using historical data, and then the estimated distribution is replicated. Therefore, it is difficult for the distribution replication approach to replicate 
a hedge fund whose return distribution substantially changes with time. For the purpose of the replication for such hedge fund strategies, a factor-based or rule-based method should be tried first. The factor-based approach is often used for the all strategies hedge fund index (see Tuchschmid et al. (2010, 2012) for example). Takahashi and Yamamoto (2009) replicated the Asian long/short equities strategy using a factor-based method. For the replication of the fixed income arbitrage strategy, the rule-based approach in Duarte et al. (2010) would be helpful.

First, the dynamic replicating strategy is applied to the replication of the managed futures index because its return distribution does not change much historically. The application to the managed futures strategy is meaningful because the factor-based approach does not work for this strategy. As the managed futures funds change market risk exposures dynamically, it is difficult to find static factors that explain the return of this strategy (see, for example, Hakamada et al. (2007)). To examine the effectiveness of the new replication method, the performance of the replication for the multi-strategy index is also studied. Finally, the replication result for the global macro index is shown to demonstrate the difficulty of replicating a hedge fund whose return distribution suffers substantial changes during a time series.

The distributions of the log returns on the hedge fund indices during the second period (2001-2011) are replicated monthly. For the purpose of their estimations, the return data in the first period (1995-2000) are also used. In this study, a Japanese investor is assumed, and the replicating strategies are based in JPY. However, the hedge fund index is denominated by USD. When the performances of replicating strategies are compared to their replication target index, the returns of the replication strategies are converted into USD with currency hedging (this means that the interest difference between USD and JPY is added to the returns of the replicating strategies). In the following out-of-sample simulation, it is assumed that the management fee for the replicating strategies is $1 \%$ per year.

Assume that the investor's existing portfolio is composed of $50 \%$ Japanese bonds and $50 \%$ Japanese stocks, which can be approximated by the equally weighted portfolio of TOPIX and Japanese government bonds (JGBs). The futures contracts on TOPIX and long-term JGB are listed on the Tokyo Stock Exchange. Replicating strategies utilize the equally weighted portfolio of these contracts as a proxy for the investor's existing portfolio, $S^{1}$.

As replicating instruments, we used the following products: the currency pairs USD/JPY, EUR/JPY, GBP/JPY, CHF/JPY, and AUD/JPY; 
the long-term JGB, US 10 year treasury note, TOPIX and S\&P500 futures as financial futures contracts; WTI crude oil futures as a commodity futures contract; JPY-denominated gold; and Libor rates as risk-free rates. Replicating strategies roll front futures contracts on the last trading day.

The transaction costs are assumed to be one basis point for the short and long of all trading instruments. All of the daily market price data are obtained from Bloomberg. The statistics of the monthly log returns on these contracts are exhibited in Table 5. USD-denominated contracts are converted into JPY because the base currency of the replicating strategies is JPY.

\subsection{Strategy Descriptions}

As mentioned in the previous subsection, managed futures, multi-strategy and global macro indices are replicated. Their strategy descriptions are as follows.

\subsubsection{Managed Futures}

Managed futures funds (often referred to as CTAs or Commodity Trading Advisors) typically focus on investing globally in listed bond, equity, commodity futures and currency markets. Managed futures fund managers tend to employ systematic trading programs that largely rely upon historical price data and market trends. A significant amount of leverage is often employed as the strategy involves the use of futures contracts. In general, CTAs do not have a particular bias towards bull or bear for any particular market. In the months immediately following the bankruptcy of Lehman Brothers, the managed futures funds achieved high returns by taking short positions on the futures contracts of risky assets because very strong downward trends appeared in the risky asset markets all over the world.

\subsubsection{Multi-Strategy}

Multi-strategy funds typically are characterized by their ability to allocate capital based upon perceived opportunities among several hedge fund strategies. Through the diversification of capital, managers seek to deliver consistently positive returns regardless of the directional movement in the equity, interest rate, or currency markets. The strategies adopted in a multi-strategy fund may include convertible bond arbitrage, long/short equity, statistical arbitrage and merger arbitrage. The benefits of diversification may reduce 
the risk profile and help to smooth the returns, reduce the volatility, and decrease the asset-class and single-strategy risks. As shown in Table 4, the standard deviation of the return is very small, and a high return-to-risk ratio is attained as compared to those of single strategies.

\subsubsection{Global Macro}

Global macro funds typically focus on identifying extreme price valuations and often take leverage against the anticipated price movements in the equity, currency, interest rate, and commodity markets. Managers typically employ a top-down global approach to concentrate on forecasting how political trends and global macroeconomic events affect the valuation of financial instruments. Profits can be made by correctly anticipating price movements in global markets and having flexibility in a broad investment mandate with the ability to hold positions in any market with any instrument. They may utilize systematic trend following models and/or discretionary judgment. Table 4 indicates that the return distribution of this strategy index has changed substantially with time. For example, the standard deviation calculated using the return data from 1995 to 2000 is $3.06 \%$, while the one calculated using the return data from 2001 to 2011 is $1.51 \%$. The performance of the global macro index from 2001 to 2011 is the best among the Dow Jones Credit Suisse Hedge Fund Indices. It attained the highest return with the lowest standard deviation.

\subsection{Empirical Methodologies}

This subsection describes the statistical methods applied in this paper. While in-sample and out-of-sample empirical studies are performed in empirical finance, replication performance with an out-of-sample basis is more important for the purpose of practical hedge fund replication. Hence, the results of only out-of-sample simulations are reported. ${ }^{1}$

Out-of-sample replications are implemented from January 2001 to December 2011 on a month-to-month basis. Monthly log return distributions are replicated through daily dynamic portfolio strategies. The replicating strategies for the marginal distribution (7) and joint distribution with the investor's existing portfolio (9) are implemented. For comparative purposes,

\footnotetext{
${ }^{1}$ The results of in-sample studies are given upon request.
} 
the replicating strategy for the joint distribution by a single instrument is also applied, which is the delta-hedging strategy for a target payoff (1).

The theory in the previous section is developed under the assumption that a target payoff distribution is given, but no one knows the true return distributions of hedge fund indices. To implement replicating strategies in practice, they should be estimated by utilizing historical data. This paper examines the impact on the performance of replicating strategies when different distribution models are used. As hedge fund returns exhibit skews and fat tails, the two different types of generalization of Gaussian distribution are considered with the aim of modeling higher moments. The first are hyperbolic and normal inverse Gaussian (NIG) distributions, which are subclasses of the generalized hyperbolic distribution class. The second are Gaussian mixture distributions.

The generalized hyperbolic distribution (GH) class is often used in financial data modeling (see, for example, Mcneil et al. (2005) or Hellmich and Kassberger (2011)). Its density function is given by

$$
g h(x ; \lambda, \alpha, \beta, \delta, \mu)=\begin{gathered}
a(\lambda, \alpha, \beta, \delta, \mu)\left\{\delta^{2}+(x-\mu)^{2}\right\}^{\lambda / 2-1 / 4} \\
\times K_{\lambda-1 / 2}\left(\alpha \sqrt{\delta^{2}+(x-\mu)^{2}}\right) \exp \{\beta(x-\mu)\},
\end{gathered}
$$

where

$$
a(\lambda, \alpha, \beta, \delta, \mu)=\frac{\left(\alpha^{2}-\beta^{2}\right)^{\lambda / 2}}{\sqrt{2 \pi} \alpha^{\lambda-1 / 2} \delta^{\lambda} K_{\lambda}\left(\delta \sqrt{\alpha^{2}-\beta^{2}}\right)}
$$

and $K_{\lambda}(x)$ is the modified Bessel function of the third kind with index $\lambda$. The parameters $(\alpha, \beta, \delta)$ are assumed to satisfy $\delta>0$ and $|\beta| \leq \alpha$ if $\lambda<0$; $\delta>0$ and $|\beta|<\alpha$ if $\lambda=0$; and $\delta \geq 0$ and $|\beta|<\alpha$ if $\lambda>0$. For $\lambda=1$, one arrives at the hyperbolic distribution. For $\lambda=-1 / 2$, one achieves the NIG distribution. For $\alpha \rightarrow \infty, \delta \rightarrow \infty$, and $\delta / \alpha \rightarrow \sigma^{2}$, one obtains a Gaussian distribution with mean $\mu+\beta \sigma^{2}$ and variance $\sigma^{2}$. In the case of the GH distribution class, the return data are fitted to Gaussian, hyperbolic and normal inverse Gaussian distributions by maximum likelihood. The bestfitted model is then selected by Akaike's information criterion (AIC).

The Gaussian mixture distribution class is used in Papageorgiou et al. (2008). The probability density function of the Gaussian mixture with $m$ regimes $(\mathrm{GM}(m))$ is given by

$$
\operatorname{gm}\left(x ; m,\left\{\pi_{k}\right\}_{k=1}^{m},\left\{\mu_{k}\right\}_{k=1}^{m},\left\{\sigma_{k}^{2}\right\}_{k=1}^{m}\right)=\sum_{k=1}^{m} \pi_{k} \phi\left(x ; \mu_{k}, \sigma_{k}^{2}\right),
$$


where $\phi\left(x ; \mu, \sigma^{2}\right)$ is the density function of the Gaussian distribution with mean $\mu$ and variance $\sigma^{2}$. In the case of Gaussian mixture distributions, the return data are fitted to $\operatorname{GM}(m)(m=1,2,3,4,5)$ by an EM-algorithm. Then, the best-fitted model is selected by AIC.

For the replication of the joint distribution with the investor's existing portfolio, the dependence structure between the target hedge fund index and the investor's existing portfolio should be modeled and estimated. Copula is applied for modeling flexible dependence structures. For example, the Clayton copula has more dependence in the lower tail than in the upper tail. This allows for the copula to capture the strong dependence in bear markets and the weak dependence in bull markets. For example, Mitchell and Pulvino (2005) showed that risk arbitrage hedge funds tend to exhibit a similar dependence structure. See, for example, Joe (1997) or Nelsen (1999) for the introduction of copula. The best-fitted copula is selected from Gaussian, Clayton, Gumbel, and Frank copulas using maximum likelihood.

\subsection{Out-of-Sample Simulation}

Let us replicate the log return distributions of the three indices on an out-ofsample basis. The monthly updated marginal distribution and joint distribution with the investor's portfolio are estimated monthly by the procedure described in the previous subsection. The last five years of data that are available at the start of the month are used for the estimations.

First, consider a replication for managed futures index. For this strategy, the currency pair USD/JPY, WTI crude oil and S\&P 500 futures contracts, and JPY-denominated gold are used as replicating instruments. We consider that managed futures fund managers trade these instruments in practice. The stochastic processes for these asset prices should be estimated. Setting the coefficients to be constants during each month, we assume that they follow log-normal processes. The parameters are updated monthly where we use an exponentially weighted moving average method. This method can reflect market trends in the parameters of the trading assets. Most managed futures fund managers analyze market trends and take positions to follow the market trends in practice. Let $\left\{r_{n}^{i}\right\}_{n=1}^{N}$ be the time-series data of the daily $\log$ return of asset $i$. Suppose that $r_{1}^{i}$ is the last sample data and $r_{N}^{i}$ is the 
oldest sample. The variance of asset $i$ is estimated by

$$
\operatorname{Var}\left(r^{i}\right)=\sum_{k=1}^{N} \frac{\lambda^{k-1}}{\sum_{n=1}^{N} \lambda^{n-1}} r_{k}^{i^{2}} .
$$

The covariances are estimated in the same manner. The drift coefficient $\mu^{i}$ is estimated by

$$
\mu^{i}=\sum_{k=1}^{N} \frac{\lambda^{k-1}}{\sum_{n=1}^{N} \lambda^{n-1}} r_{k}^{i}+\frac{1}{2} \operatorname{Var}\left(r^{i}\right) .
$$

Here, the decay factor $\lambda$ is assumed to be 0.99 . The half value period is then 68 days.

Replicating strategies that utilize GH and GM distribution classes are implemented. For comparative purposes, the replicating strategy of a single replicating instrument for the joint distribution is also applied, where the replicating instrument is the equally weighted portfolio of the four instruments used in this replication. Table 1 exhibits the summary statistics of the log returns of the managed futures index and its replicating strategies.

The replicating strategies with the GH distribution class attained higher returns than they did with the GM distributions. The following two reasons for this difference can be considered. First, a Gaussian mixture distribution has an over-fitting problem as it may have many free parameters. In other words, it is not robust to out-of-sample predictions but is able to fit the model to in-sample data. Second, the replicating strategy for a distribution whose density function has multiple peaks does not seem to be reasonable as an investment strategy. $f^{\prime}\left(L_{T}\right)$ in equation (8) is represented as $f^{\prime}\left(L_{T}\right)=\phi_{L_{T}}\left(L_{T}\right) / \phi_{\xi}\left(f\left(L_{T}\right)\right)$, where $\phi_{L_{T}}$ and $\phi_{\xi}$ are the density functions for $L_{T}$ and $\xi$, respectively. This means that the gross position amount is determined by the density function of the target distribution. If the density of the target distribution has multiple peaks, then the gross position amount of the replicating strategy can change substantially with the state of $L_{t}$. Although the replicating strategy can regenerate such a multi-peak return histogram, it is doubtful that such a dynamic strategy is reasonable or superior for investment.

Figure 1 shows the net asset values of the managed futures index and replicating strategies with the $\mathrm{GH}$ distribution class. The replicating strategies with multiple instruments performed very well. Although the replicating strategy with a single replicating instrument outperformed the managed 
Table 1: Summary Statistics on the Log Returns of the Managed Futures Index and Its Replicating Strategies (2001-2011)

\begin{tabular}{ccccc}
\hline & $\begin{array}{c}\text { Managed Futures } \\
\text { Index }\end{array}$ & $\begin{array}{c}\text { Multiple } \\
\text { Marginal }\end{array}$ & $\begin{array}{c}\text { Multiple } \\
\text { Joint }\end{array}$ & $\begin{array}{c}\text { Single } \\
\text { Joint }\end{array}$ \\
\hline Mean & $0.52 \%$ & $0.89 \%$ & $0.87 \%$ & $0.61 \%$ \\
Std. Dev. & $3.45 \%$ & $3.61 \%$ & $3.49 \%$ & $2.92 \%$ \\
Mean/Std. Dev & 0.15 & 0.25 & 0.25 & 0.21 \\
Skew & -0.15 & 0.33 & 0.20 & -0.61 \\
Kurtosis & 2.37 & 2.95 & 2.77 & 5.10 \\
Max & $8.28 \%$ & $12.03 \%$ & $9.82 \%$ & $6.92 \%$ \\
Min & $-9.02 \%$ & $-7.49 \%$ & $-7.59 \%$ & $-12.46 \%$ \\
Correlation with & -0.03 & 0.07 & 0.03 & 0.34 \\
the investor's portfolio & & & & \\
Correlation with & & 0.57 & 0.56 & 0.11 \\
the target index & & & & \\
\hline
\end{tabular}

(a) The Generalized Hyperbolic Class

\begin{tabular}{ccccc}
\hline & $\begin{array}{c}\text { Managed Futures } \\
\text { Index }\end{array}$ & $\begin{array}{c}\text { Multiple } \\
\text { Marginal }\end{array}$ & $\begin{array}{c}\text { Multiple } \\
\text { Joint }\end{array}$ & $\begin{array}{c}\text { Single } \\
\text { Joint }\end{array}$ \\
\hline Mean & $0.52 \%$ & $0.86 \%$ & $0.66 \%$ & $0.59 \%$ \\
Std. Dev. & $3.45 \%$ & $3.63 \%$ & $3.49 \%$ & $2.97 \%$ \\
Mean/Std. Dev & 0.15 & 0.24 & 0.19 & 0.20 \\
Skew & -0.15 & 0.26 & 0.07 & -0.56 \\
Kurtosis & 2.37 & 2.83 & 2.65 & 4.04 \\
Max & $8.28 \%$ & $12.03 \%$ & $9.67 \%$ & $6.92 \%$ \\
Min & $-9.02 \%$ & $-7.43 \%$ & $-7.86 \%$ & $-11.32 \%$ \\
Correlation with & -0.03 & 0.07 & 0.00 & 0.34 \\
the investor's portfolio & & & & \\
Correlation with & & 0.57 & 0.57 & 0.12 \\
the target index & & &
\end{tabular}

(b) Gausiann Mixture Distributions 
futures index, it was not able to replicate an attractive character of the managed futures strategy: good performance under strong bear markets. Especially in the months immediately following the bankruptcy of Lehman Brothers, managed futures funds achieved high returns by taking short positions on the futures contracts of risky assets because very strong downward trends appeared in the risky asset markets all over the world. Replication with a single instrument incurred a large loss during this period because it cannot take short position on the instrument. However, replications with multiple replicating instruments succeeded in replicating good performance under the strong bear market by taking short positions on risky assets. As shown in Table 1, replicating strategies with multiple instruments have higher correlations with the managed futures index than do those with a single instrument.

Figure 1: The net asst vales of the managed futures index and its replicating strategies with the GH distribution class (Jan 2001-Dec 2011).

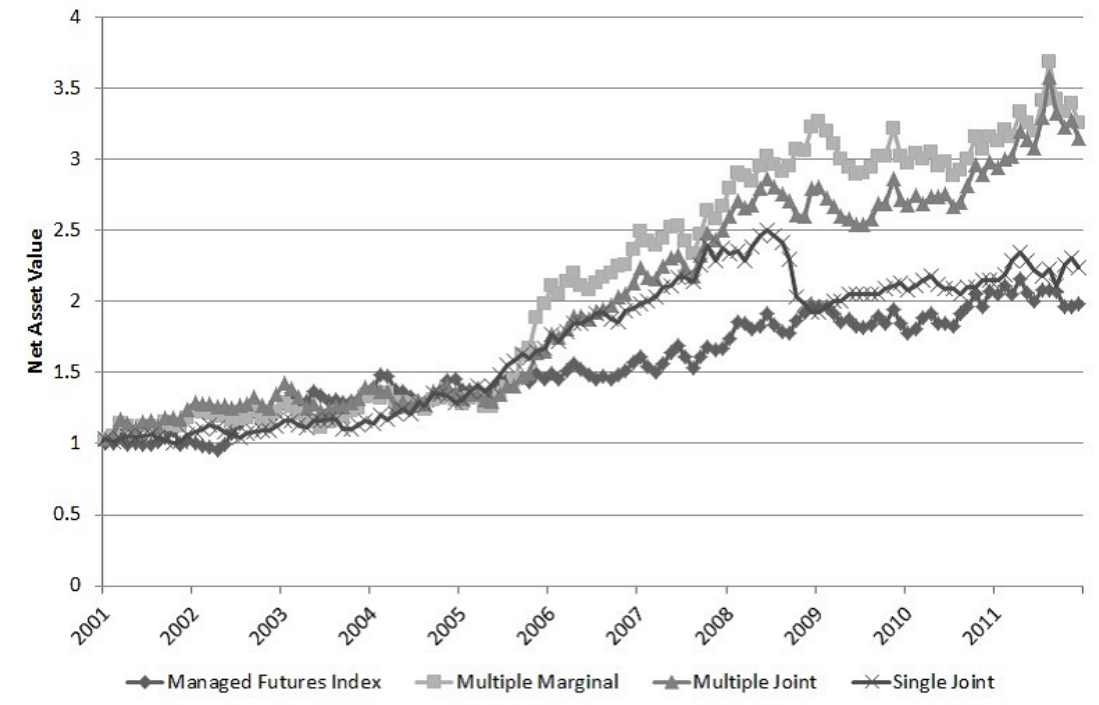

Next, the replication for the multi-strategy index is performed. For this replication, all of the foreign exchange and futures contracts listed in subsection 4.1 are used as replicating instruments. They are put into an equally weighted portfolio that is used for the replication with a single instrument. In the estimation procedure for the trading instruments, the decay factor $\lambda$ 
is assumed to be 0.9975 . Other procedures are the same as in the replications for the managed futures. Table 2 shows the summary statistics of the replication results. The replications with the $\mathrm{GH}$ distribution class obtained higher mean returns than did those with GM distributions for this case, as well.

Table 2: Summary Statistics on the Log Returns of the Multi-Strategy Index and Its Replicating Strategies (2001-2011)

\begin{tabular}{ccccc}
\hline & $\begin{array}{c}\text { Multi-Strategy } \\
\text { Index }\end{array}$ & $\begin{array}{c}\text { Multiple } \\
\text { Marginal }\end{array}$ & $\begin{array}{c}\text { Multiple } \\
\text { Joint }\end{array}$ & $\begin{array}{c}\text { Single } \\
\text { Joint }\end{array}$ \\
\hline Mean & $0.52 \%$ & $0.49 \%$ & $0.55 \%$ & $0.30 \%$ \\
Std. Dev. & $1.62 \%$ & $1.94 \%$ & $1.72 \%$ & $1.16 \%$ \\
Mean/Std. Dev & 0.32 & 0.25 & 0.32 & 0.26 \\
Skew & -2.03 & -0.28 & 0.37 & -0.75 \\
Kurtosis & 11.21 & 6.70 & 5.64 & 5.30 \\
Max & $4.19 \%$ & $7.74 \%$ & $7.41 \%$ & $2.98 \%$ \\
Min & $-7.63 \%$ & $-7.55 \%$ & $-4.20 \%$ & $-4.56 \%$ \\
Correlation with & 0.54 & 0.15 & 0.16 & 0.55 \\
the investor's portfolio & & & & \\
Correlation with & & 0.20 & 0.10 & 0.53 \\
the target index & & & &
\end{tabular}

(a) Generalized Hyperbolic Class

\begin{tabular}{ccccc}
\hline & $\begin{array}{c}\text { Multi-Strategy } \\
\text { Index }\end{array}$ & $\begin{array}{c}\text { Multiple } \\
\text { Marginal }\end{array}$ & $\begin{array}{c}\text { Multiple } \\
\text { Joint }\end{array}$ & $\begin{array}{c}\text { Single } \\
\text { Joint }\end{array}$ \\
\hline Mean & $0.52 \%$ & $0.37 \%$ & $0.41 \%$ & $0.27 \%$ \\
Std. Dev. & $1.62 \%$ & $1.85 \%$ & $1.63 \%$ & $1.39 \%$ \\
Mean/Std. Dev & 0.32 & 0.20 & 0.25 & 0.20 \\
Skew & -2.03 & -2.02 & -2.25 & -3.91 \\
Kurtosis & 11.21 & 9.50 & 12.11 & 31.62 \\
Max & $4.19 \%$ & $4.03 \%$ & $3.71 \%$ & $2.92 \%$ \\
Min & $-7.63 \%$ & $-8.58 \%$ & $-8.80 \%$ & $-10.65 \%$ \\
Correlation with & 0.54 & 0.09 & 0.32 & 0.60 \\
the investor's portfolio & & & & \\
Correlation with & & 0.15 & 0.29 & 0.61 \\
the target index & & & \\
\hline
\end{tabular}

(b) Gaussian Mixture Distributions

Figure 2 shows the net asset values of the multi-strategy index and replicating strategies with the GH distribution class. The replication for the joint 
distribution with multiple assets outperformed the multi-strategy index. The replication for the marginal distribution slightly underperformed the multistrategy index, but it attained a close return level. The extension to multiple assets much improved the performance for this case, as well.

Figure 2: The net asst vales of the multi-strategy index and its replicating strategies with the GH distribution class (Jan 2001-Dec 2011).

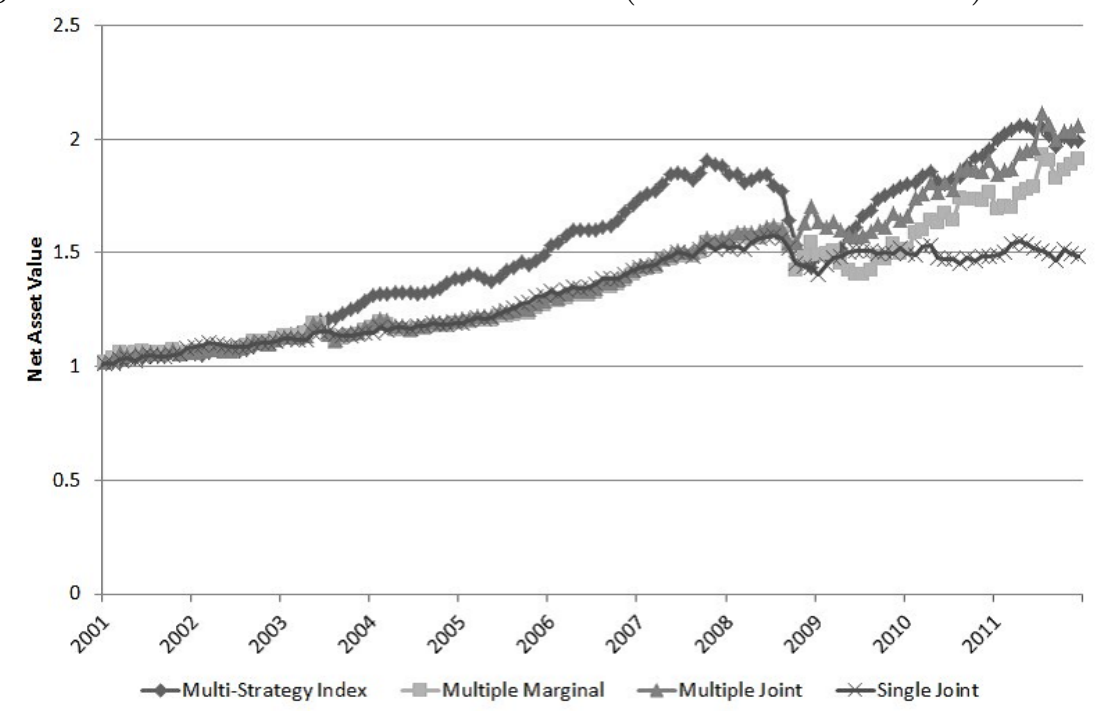

Finally, let us replicate the global macro index. In this replication, the entire procedure is exactly the same as it was in the replication for the multistrategy case. Table 3 shows the summary statistics of the replication results. The replications with the GH distribution class attained higher mean returns than did those with GM distributions for the global macro case, as well. This phenomenon is observed for all of the replications for the three strategies.

Figure 3 shows the net asset values of the global macro index and the replicating strategies for the GH distribution class. Replicating strategies could not attain returns that were as high as those of the global macro index. The following two reasons for this failure are considered. First, the return distribution of the global macro index changes substantially with time. Therefore, it is difficult to replicate it on an out-of-sample basis. Second, the performance of the global macro index during the sample period is very high. As previously mentioned, the performance of the global macro index from 
Table 3: Summary Statistics on the Log Returns of the Global Macro Index and Its Replicating Strategies (2001-2011)

\begin{tabular}{ccccc}
\hline & $\begin{array}{c}\text { Global Macro } \\
\text { Index }\end{array}$ & $\begin{array}{c}\text { Multiple } \\
\text { Marginal }\end{array}$ & $\begin{array}{c}\text { Multiple } \\
\text { Joint }\end{array}$ & $\begin{array}{c}\text { Single } \\
\text { Joint }\end{array}$ \\
\hline Mean & $0.89 \%$ & $0.63 \%$ & $0.74 \%$ & $0.42 \%$ \\
Std. Dev. & $1.51 \%$ & $2.79 \%$ & $2.71 \%$ & $2.02 \%$ \\
Mean/Std. Dev & 0.59 & 0.23 & 0.27 & 0.21 \\
Skew & -1.41 & -0.37 & 0.03 & -0.31 \\
Kurtosis & 9.21 & 5.66 & 5.49 & 7.63 \\
Max & $4.35 \%$ & $10.20 \%$ & $10.46 \%$ & $7.81 \%$ \\
Min & $-6.86 \%$ & $-9.37 \%$ & $-8.63 \%$ & $-8.99 \%$ \\
Correlation with & 0.22 & 0.14 & 0.07 & 0.39 \\
the investor's portfolio & & & & \\
Correlation with & & 0.49 & 0.41 & 0.30 \\
the target index & &
\end{tabular}

(a) The Generalized Hyperbolic Class

\begin{tabular}{ccccc}
\hline & $\begin{array}{c}\text { Global Macro } \\
\text { Index }\end{array}$ & $\begin{array}{c}\text { Multiple } \\
\text { Marginal }\end{array}$ & $\begin{array}{c}\text { Multiple } \\
\text { Joint }\end{array}$ & $\begin{array}{c}\text { Single } \\
\text { Joint }\end{array}$ \\
\hline Mean & $0.89 \%$ & $0.55 \%$ & $0.57 \%$ & $0.44 \%$ \\
Std. Dev. & $1.51 \%$ & $2.83 \%$ & $2.86 \%$ & $2.07 \%$ \\
Mean/Std. Dev & 0.59 & 0.19 & 0.20 & 0.21 \\
Skew & -1.41 & -1.00 & -0.93 & -1.68 \\
Kurtosis & 9.21 & 7.47 & 7.82 & 16.96 \\
Max & $4.35 \%$ & $10.20 \%$ & $10.46 \%$ & $7.81 \%$ \\
Min & $-6.86 \%$ & $-11.07 \%$ & $-10.87 \%$ & $-12.96 \%$ \\
Correlation with & 0.22 & 0.12 & 0.15 & 0.42 \\
the investor's portfolio & & & & \\
Correlation with & & 0.46 & 0.47 & 0.36 \\
the target index & & &
\end{tabular}

(b) Gaussian Mixture Distributions 
2001 to 2011 is the best among the Dow Jones Credit Suisse Hedge Fund Indices. Although replicating strategies with multiple instruments underperformed the global macro index, the extension to multiple assets improves the performance substantially.

Figure 3: The net asst vales of the global macro index and its replicating strategies with the GH distribution class (Jan 2001-Dec 2011).

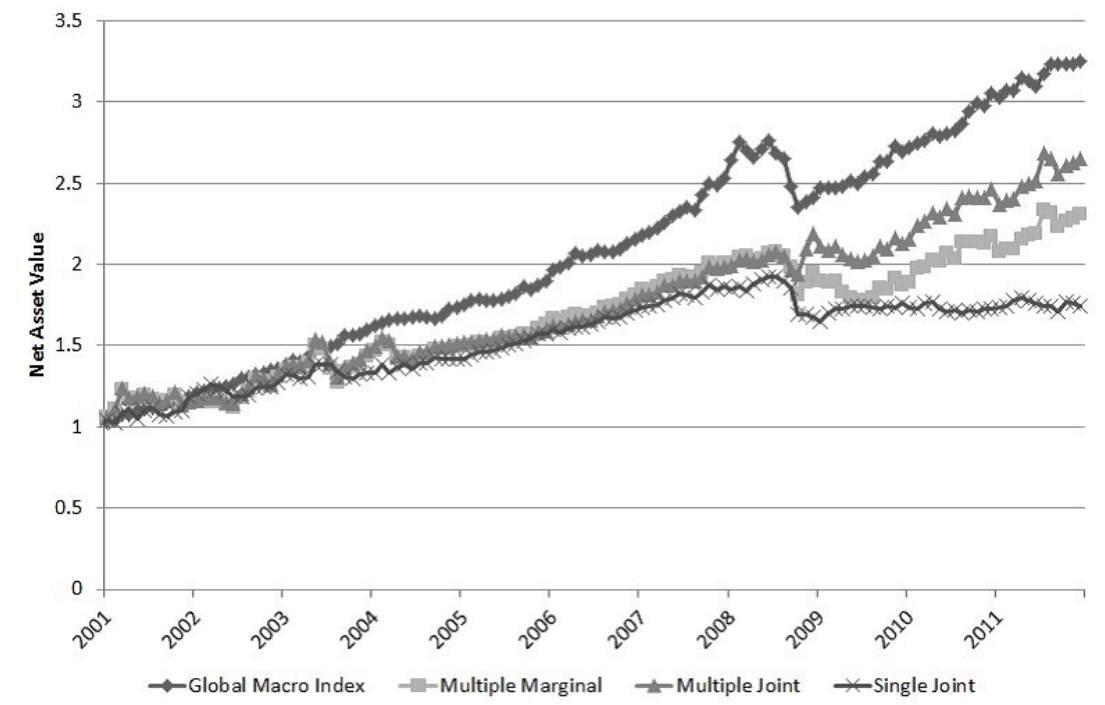

\section{Conclusion}

This article presented a new method to construct the cheapest dynamic portfolio that generates a target payoff distribution or its joint distribution with the investor's existing portfolio. It was shown that the cost minimization is equivalent to the maximization of a certain class of von NeumannMorgenstern utility functions. This method is regarded as an extension of the hedge fund replication methods that were developed by Kat and Palaro (2005a, b) and Papageorgiou, Remillard and Hocquard (2008). Their methods replicate the distribution of the target hedge fund and its dependence upon the investor's existing portfolio by trading the investor's portfolio and one replicating instrument with only long positions. Our method extends 
their approach to allow for the trading of multiple assets with both long and short positions.

Finally, the method was applied to the replications of three hedge fund strategies: managed futures, multi-strategy and global macro. The replication performances were examined on an out-of-sample basis. The results showed that the performances of the replications improved substantially as compared to the case of one replicating instrument with only long positions. Additionally, two different distribution classes, the Generalized Hyperbolic (GH) distribution class and Gaussian Mixture (GM) distributions, are applied to the estimation of the target hedge fund indices' returns; replications with the GH distribution class outperformed those with the GM distribution for all of the three strategies. As a next research topic, the implementation of the Markovian coefficients case, including a stochastic volatility model and a stochastic interest rate model, is a challenging task. Additionally, the applications of our method to creating attractive new trading strategies and risk management are interesting topics. 

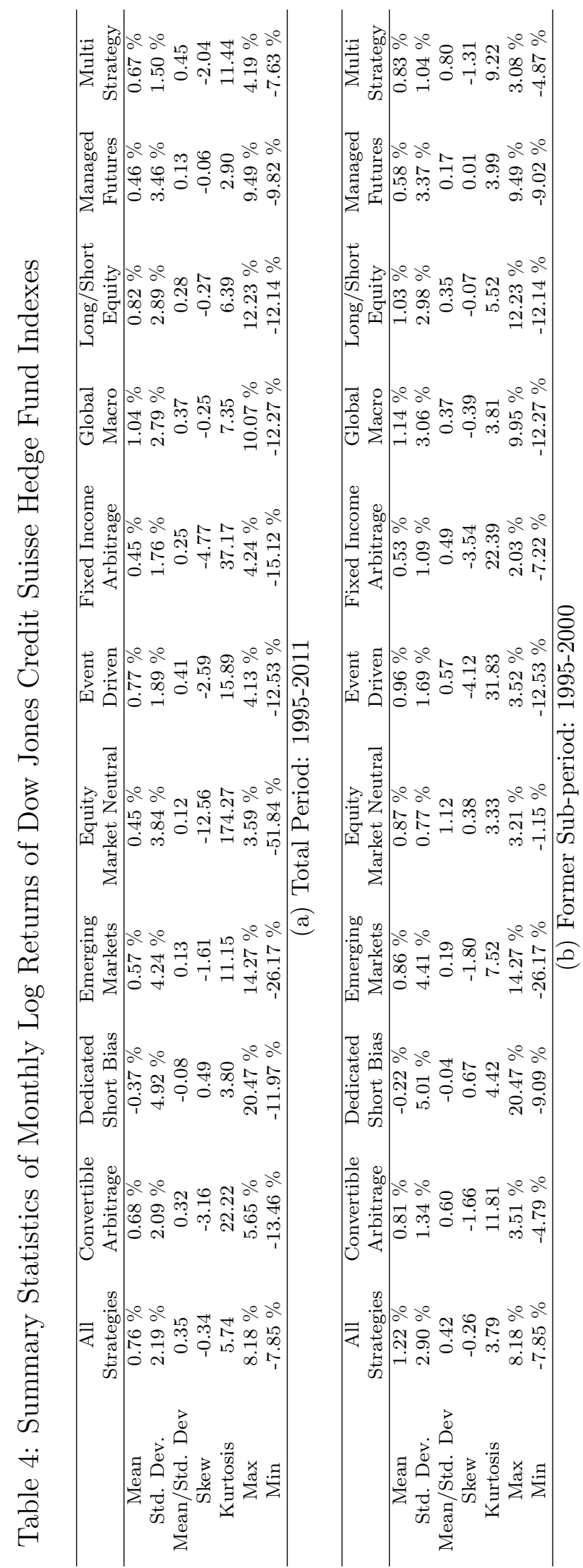

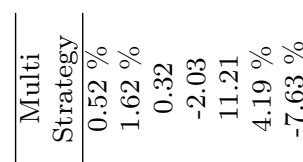

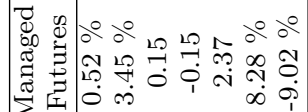

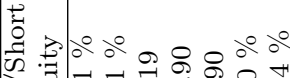

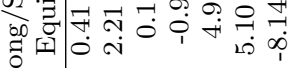

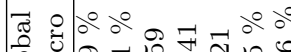
U

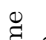

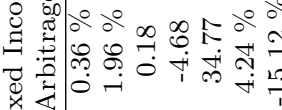

$x$

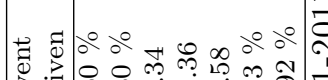

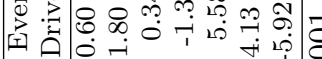

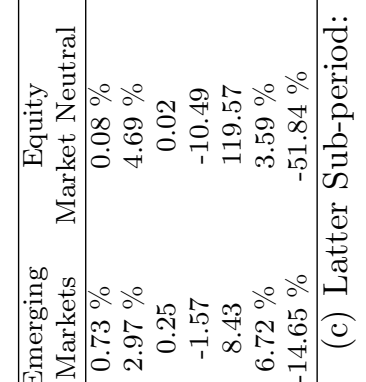

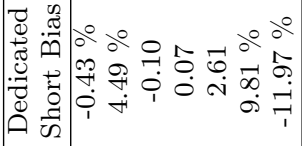

然

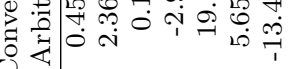

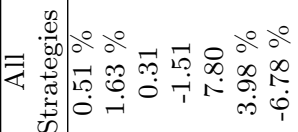

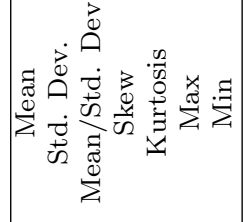




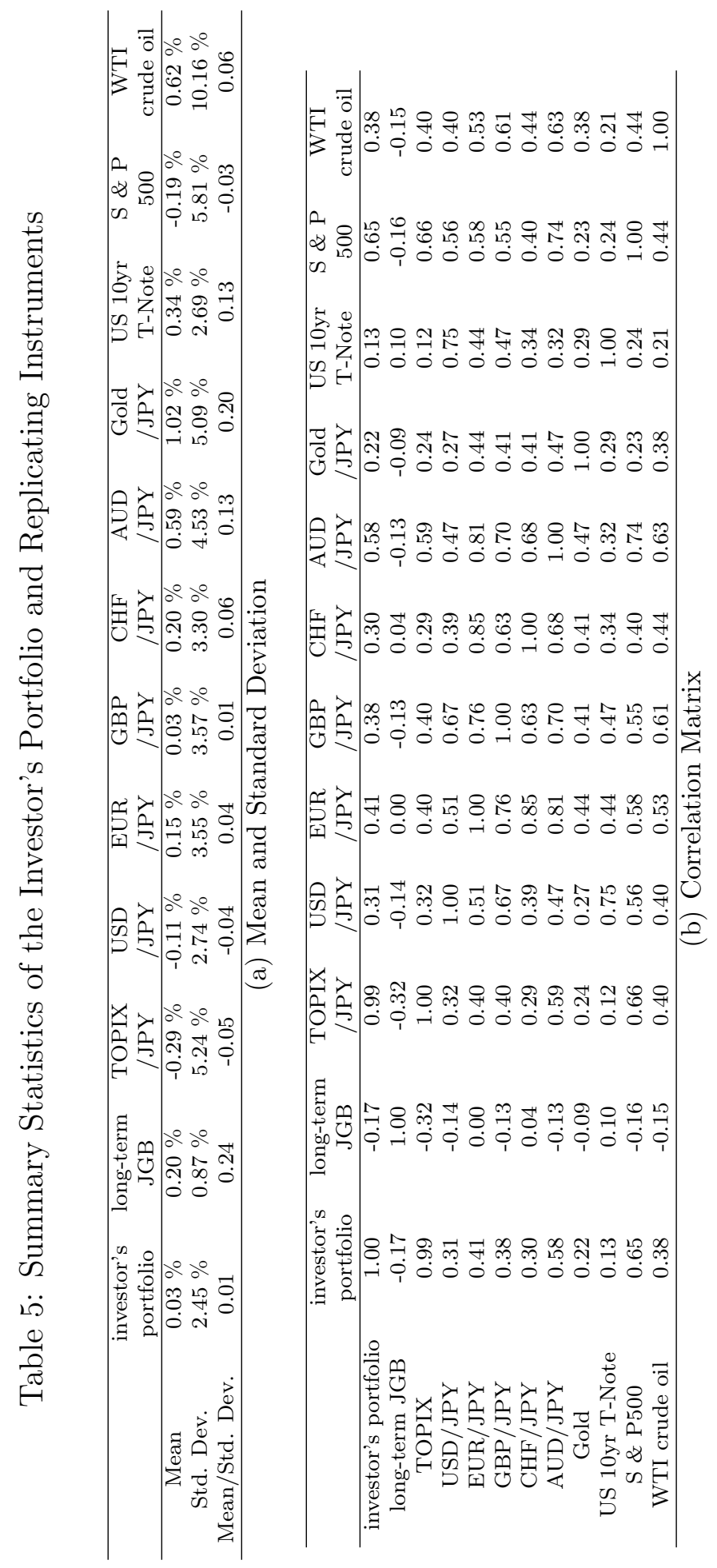




\section{References}

Agarwal, V. and N.Y. Naik (2004). Risks and Portfolio Decisions Involving Hedge Funds. The Review of Financial Studies, Vol. 17(1), pp. 63-98.

Amin, G. and H.M. Kat (2003). Hedge Fund Performance 1990-2000: Do the Money Machines Really Add Value? Journal of Financial and Quantitative Analysis, Vol. 38(2), pp. 251-274.

Cvitanić, V. Polimenis and F. Zapatero (2008). Optimal Portfolio Allocation with Higher Moments. Annals of Finance, Vol. 4, pp. 1-28.

Duarte, J., F. Longstaff, and F. Yu (2007). Risk and Return in Fixed Income Arbitrage: Nickels in front of a Steamroller? The Review of Financial Studies, Vol. 20(3), pp. 769-811.

Dybvig, P. (1988a). Distributional Analysis of Portfolio Choice. Journal of Business, Vol. 61, pp. 369-393.

Dybvig, P. (1988b). Inefficient Dynamic Portfolio Strategies or How to Throw Away a Million Dollars in the Stock Market. The Review of Financial Studies, Vol. 1, pp. 67-88.

Fung, W. and D.A. Hsieh (1997). Empirical Characteristics of Dynamic Trading Strategies: The Case of Hedge Funds. The Review of Financial Studies, Vol. 10(2), pp. 275-302.

Fung, W. and D.A. Hsieh (2000). Performance Characteristics of Hedge Funds and Commodity Funds: Natural vs. Spurious Biases. The Journal of Financial and Quantitative Analysis, Vol. 35(3), pp. 291-307.

Fung, W. and D.A. Hsieh (2001). The Risk in Hedge Fund Strategies: Theory and Evidence from Trend Followers. The Review of Financial Studies, Vol. 14(2), pp. 313-341.

Fung, W. and D.A. Hsieh (2007a). Will Hedge Funds Regress towards Indexlike Products? Journal of Investment Management, Vol. 5(2), pp. 46-55.

Fung, W. and D.A. Hsieh (2007b). Hedge Fund Replication Strategies: Implications for Investors and Regulators. Banque de France, Financial Stability Review, Special issue on hedge funds, Vol. 10 April, pp. 55-66. 
Hakamada, T., A. Takahashi, and K. Yamamoto (2007). Selection and Performance Analysis of Asia-Pacific Hedge Funds. The Journal of Alternative Investments, Vol. 10(3), pp. 7-29.

Hellmich, M. and S. Kassberger (2011). Efficient portfolio optimization the multivariate Generalized Hyperbolic framework. Quantitative Finance, Vol. 11(10), pp. 1503-1516.

Hocquard, A., N. Papageorgiou, and B. Remillard (2012) The Payoff Distribution Model: An Application to Portfolio Insurance Quantitative Finance, Forthcoming.

Joe, H. (1997). Multivariate Models and Dependence Concepts. Chapman and Hall.

Karatzas, I. and S. Shreve (1998). Methods of Mathematical Finance. Springer.

Kat, H.M. and H.P. Palaro (2005a). Who Needs Hedge Funds? A CopulaBased Approach to Hedge Fund Return Replication. Working Paper.

Kat, H.M. and H.P. Palaro (2005b). Hedge Fund Returns: You Can Make Them Yourself! Journal of Wealth Management, Vol. 8(2), pp. 62-68.

Lo, A. and J. Hasanhodzic (2007). Can Hedge-Fund Returns Be Replicated?: The Linear Case. Journal of Investment Management, Vol. 5(2), pp. 5-45.

Malkiel, B.G. and A. Saha (2005). Hedge Funds: Risk and Return. The Financial Analysts Journal, Vol. 61(6), pp. 80-88.

Markowitz, H.M. (1952). Portfolio Selection. The Journal of Finance, Vol. 7(1): pp. $77-91$

McNeil, A.J., R. Frey and P. Embrechts (2005). Quantitative Risk Management: Concepts, Techniques, and Tools. Princeton University Press.

Merton, R.C. (1969). Lifetime Portfolio Selection under Uncertainty: The continuous time case. Review of Economics 8 Statistics, Vol. 51(3), pp. $247-257$

Merton, R.C., (1971). Optimum Consumption and Portfolio Rules in a Continuous-time Model, Journal of Economic Theory, Vol. 3(4), pp. 373-413 
Mitchell, M. and T. Pulvino (2001). Characteristics of Risk in Risk Arbitrage. Journal of Finance, Vol. 56, pp. 2067-2109.

Nelsen, R.B. (1999). An introduction to copulas: Vol. 139 of Lecture Notes in Statistics. Springer-Verlag, New York.

Nualart, D. (2006). The Malliavin Calculus and Related Topics 2nd Edition, Springer.

Ocone, D., and I. Karatzas (1991). A Generalized Clark Representation Formula, with Applications to Optimal Portfolios. Stochastics, Vol. 37, pp. 187-220.

Papageorgiou, N., B. Remillard, and A. Hocquard (2008). Replicating the Properties of Hedge Fund Returns. The Journal of Alternative Investments, Vol. 11(2), pp. 8-38.

Takahashi, A., and K. Yamamoto (2010). Hedge Fund Replication. Chapter 2 in The Recent Trend of Hedge Fund Strategies, Nova Science Publishers, pp. 57-95.

Takahashi, A., and K. Yamamoto (2010). A New Hedge Fund Replication Method with the Dynamic Optimal Portfolio. Global Journal of Business Research, Vol. 4(4), pp. 23-34.

Takahashi, A., and N. Yoshida (2004). An Asymptotic Expansion Scheme for Optimal Investment Problems. Statistical Inference for Stochastic Process, Vol. 7, pp. 153-188.

Tuchschmid, N.S., E. Wallerstein and S. Zaker (2010). How Do Hedge Fund Clones Manage the Real World? The Journal of Alternative Investments, Vol. 12(3), pp. 37-50.

Tuchschmid, N.S., E. Wallerstein and S. Zaker (2012). Hedge Fund Clones, Chapter 8 in The Oxford Handbook of Quantitative Asset Management, Oxford University Press, pp. 136-153. 


\section{Appendix}

\subsection{Proof of Theorem 1}

First, let us show that the cheapest way to obtain a target distribution is achieved by allocating the terminal wealth in the reverse order of the state price density. The reverse order of the state price density is mathematically defined as follows. Let $Z$ be a random variable. For $\omega^{*} \in \Omega$, define $U_{Z}\left(\omega^{*}\right)$ as

$$
U_{Z}\left(\omega^{*}\right)=\left\{\omega: H_{T}(\omega)<H_{T}\left(\omega^{*}\right)\right\} \cap\left\{\omega: Z(\omega)<Z\left(\omega^{*}\right)\right\}
$$

and let

$$
V_{Z}=\cup_{\omega^{*} \in \Omega} U_{Z}\left(\omega^{*}\right) .
$$

$Z$ is the reverse order of the state price density if

$$
\mathbb{P}\left(V_{Z}\right)=0 .
$$

Assume that the $\mathcal{F}_{T}$-measurable random variable $Z$ has the same distribution as $\xi$, and is not the reverse order of the state price density. Then, there exist $a_{1}^{-}<a_{1}^{+} \leq a_{2}^{-}<a_{2}^{+}$, such that

$$
H_{T}\left(\omega_{1}\right)<H_{T}\left(\omega_{2}\right) \text { for any }\left(\omega_{1}, \omega_{2}\right) \in A_{1} \times A_{2},
$$

where $A_{1}=\left\{a_{1}^{-}<Z<a_{1}^{+}\right\}, A_{2}=\left\{a_{2}^{-}<Z<a_{2}^{+}\right\}$, and $\mathbb{P}\left(A_{1}\right)=\mathbb{P}\left(A_{2}\right)>0$. $Z$ is not the reverse order of $H_{T}$ on $A_{1} \cup A_{2}$.

The following discussion shows that a payoff cheaper than $Z$ can be created without changing the distribution by switching the values on $A_{1}$ and $A_{2}$. Let $p_{i}^{ \pm}=\mathbb{P}\left(Z \leq a_{i}^{ \pm}\right), I_{1}=\left(p_{1}^{-}, p_{1}^{+}\right]$, and $I_{2}=\left(p_{2}^{-}, p_{2}^{+}\right]$. Define $Z^{\prime}$ as

$$
Z^{\prime}=G\left(F_{\xi}(Z)\right)
$$

where function $G$ is defined as

$$
G(p)= \begin{cases}F_{\xi}^{-1}(p) \quad \text { on }(0,1) \backslash & \left(I_{1} \cup I_{2}\right), \\ F_{\xi}^{-1}\left(p+p_{2}^{-}-p_{1}^{-}\right) & \text {on } I_{1}, \\ F_{\xi}^{-1}\left(p+p_{1}^{-}-p_{2}^{-}\right) & \text {on } I_{2} .\end{cases}
$$

Here, note that $p_{1}^{+}-p_{1}^{-}=p_{2}^{+}-p_{2}^{-}$. Then, $Z^{\prime}$ has the same distribution as $\xi$ because the equation

$$
\mathbb{P}\left(Z^{\prime} \leq a\right)=F_{\xi}(a)
$$


holds true for any $a>0$, as shown in the following. For $0<a \leq a_{1}^{-}$,

$$
\mathbb{P}\left(Z^{\prime} \leq a\right)=F_{\xi}(a)
$$

For $a \in\left(a_{1}^{-}, a_{1}^{+}\right]$,

$$
\begin{aligned}
\mathbb{P}\left(Z^{\prime} \leq a\right) & =\mathbb{P}\left(Z^{\prime} \leq a_{1}^{-}\right)+\mathbb{P}\left(a_{1}^{-}<Z^{\prime} \leq a\right) \\
& =\mathbb{P}\left(Z \leq a_{1}^{-}\right)+\mathbb{P}\left(a_{1}^{-}<G\left(F_{\xi}(Z)\right) \leq a\right) \\
& =p_{1}^{-}+\mathbb{P}\left(F_{\xi}\left(a_{1}^{-}\right)<F_{\xi}(Z)+p_{2}^{-}-p_{1}^{-} \leq F_{\xi}(a)\right) \\
& =F_{\xi}(a) .
\end{aligned}
$$

The same arguments prove that equation (13) holds true for any $a$ in the other intervals.

The difference in the costs is given by

$$
E\left[H_{T} Z\right]-E\left[H_{T} Z^{\prime}\right]=E\left[H_{T}\left(Z-Z^{\prime}\right) 1_{A_{2}}\right]-E\left[H_{T}\left(Z^{\prime}-Z\right) 1_{A_{1}}\right] .
$$

As $Z^{\prime}$ is created by switching the values of $Z^{\prime}$ on $A_{1}$ and $A_{2},\left(Z-Z^{\prime}\right) 1_{A_{2}}$ and $\left(Z^{\prime}-Z\right) 1_{A_{1}}$ have the same distribution. Noting inequality (12),

$$
E\left[H_{T} Z\right]-E\left[H_{T} Z^{\prime}\right]>0 .
$$

To summarize, $Z^{\prime}$ has the same distribution as $\xi$ with a cheaper cost than $Z$. Therefore, to obtain the same distribution as $\xi$ at time $T$, the terminal wealth should be in the reverse order of the state price density. Because $X$ is the reverse order of $H_{T}, X$ is one of the cheapest payoffs that has the same distribution as $\xi$.

Next, let us prove the uniqueness. Let $X^{\prime}$ be a random variable that has the same distribution as $\xi$, and is the reverse order of $H_{T}$. Then, $P\left(V_{X^{\prime}}\right)=0$. For any $\omega^{*} \in \Omega \backslash V_{X^{\prime}}$,

$$
\mathbb{P}\left\{\omega: L_{T}(\omega) \leq L_{T}\left(\omega^{*}\right)\right\}=\mathbb{P}\left\{\omega: X^{\prime}(\omega) \leq X^{\prime}\left(\omega^{*}\right)\right\} .
$$

Therefore,

$$
F_{L_{T}}\left(L_{T}\left(\omega^{*}\right)\right)=F_{\xi}\left(X^{\prime}\left(\omega^{*}\right)\right) .
$$

By operating $F_{\xi}^{-1}$ to the both sides of the equation,

$$
F_{\xi}^{-1}\left(F_{L_{T}}\left(L_{T}\left(\omega^{*}\right)\right)\right)=X^{\prime}\left(\omega^{*}\right) .
$$

Hence, $X^{\prime}=X$. 


\subsection{Proof of Theorem 2}

Let $x$ be the initial cost for the payoff $X$ (i.e., $x=E\left[H_{T} X\right]$ ). As $X$ is defined by equation (5), $H_{T}=\exp \left[-f^{-1}(X)\right] \cdot \exp \left[-f^{-1}(\cdot)\right]$ is a positive, strictly decreasing function. Define $u(\cdot)$ as

$$
u(z)=\lambda \int_{0}^{z} \exp \left[-f^{-1}(\zeta)\right] d \zeta
$$

where $\lambda$ is a positive number. Then, $u(\cdot)$ is strictly increasing and strictly concave. It is also true that $\lim _{z \rightarrow+0} u^{\prime}(z)=+\infty$ and $\lim _{z \rightarrow+\infty} u^{\prime}(z)=0$. Moreover, $u^{\prime}(X)=\lambda H_{T}$. This is the first order condition of the optimality for von Neumann-Morgenstern utility $u(\cdot)$ (see, for example, Karatzas and Shreve (1998)). The budget constraint $x=E\left[H_{T} u^{\prime-1}\left(\lambda H_{T}\right)\right]$ is also satisfied.

Conversely, assume a dynamic trading strategy with the initial cost $x^{\prime}$, generating payoff the $X^{\prime}$ maximizes a strictly increasing and strictly concave von Neumann-Morgenstern utility $u(\cdot)$ satisfying conditions (a) and (b). Then, $u^{\prime}\left(X^{\prime}\right)=\lambda^{\prime} H_{T}$ is satisfied for some $\lambda^{\prime}>0$. Therefore, $X^{\prime}$ is the reverse order of $H_{T}$. According to the argument in the proof of Theorem 1, this strategy attains the cheapest payoff among the random variables whose distribution is the same as $X^{\prime}$.

\subsection{Proof of Theorem 3}

The basic idea is the same as the proof of theorem 1 . Let $Z$ be a random variable whose joint distributions with $S_{T}^{1}$ is the same as $\left(S_{T}^{1}, \xi\right)$. Define $\mathcal{S}_{Z} \subset \mathbb{R}_{+}$and $B_{Z}^{1} \subset \Omega$ as

$$
\mathcal{S}_{Z}=\left\{s: Z \text { is not reverse order of } H_{T} \text { under the condition } S_{T}^{1}=s\right\},
$$

$$
B_{Z}^{1}=\left\{\omega: S_{T}^{1}(\omega) \in \mathcal{S}_{Z}\right\}
$$

Assume that $\mathbb{P}\left(B_{Z}^{1}\right)>0$. For any $s \in \mathcal{S}_{Z}$, there exist $a_{1}^{s-}<a_{1}^{s+} \leq a_{2}^{s-}<a_{2}^{s+}$, such that

$$
H_{T}\left(\omega_{1}\right)<H_{T}\left(\omega_{2}\right) \text { for any }\left(\omega_{1}, \omega_{2}\right) \in A_{1}^{s} \times A_{2}^{s},
$$

where $A_{1}^{s}=\left\{S_{T}^{1}=s, a_{1}^{s-}<Z<a_{1}^{s+}\right\}, A_{2}^{s}=\left\{S_{T}^{1}=s, a_{2}^{s-}<Z<a_{2}^{s+}\right\}$, and $\mathbb{P}\left(A_{1}^{s} \mid S_{T}^{1}=s\right)=\mathbb{P}\left(A_{2}^{s} \mid S_{T}^{1}=s\right)>0$. Let $p_{i}^{s \pm}=\mathbb{P}\left(Z \leq a_{i}^{s \pm} \mid S_{T}^{1}=s\right)$, $I_{1}^{s}=\left(p_{1}^{s-}, p_{1}^{s+}\right]$ and $I_{2}^{s}=\left(p_{2}^{s-}, p_{2}^{s+}\right]$. Introduce random variable $Z^{\prime}$, defined by

$$
Z^{\prime}=\left\{\begin{array}{l}
G^{1}\left(S_{T}^{1}, F_{\xi \mid S_{T}^{1}}(Z)\right) \\
Z \quad \text { on } \Omega \backslash B_{Z}^{1},
\end{array}\right.
$$


where $G^{1}$ is defined as

$$
G^{1}(s, p)= \begin{cases}F_{\xi \mid s}^{-1}(p) \quad \text { for } p \in(0,1) \backslash\left(I_{1}^{s} \cup I_{2}^{s}\right), \\ F_{\xi \mid s}^{-1}\left(p+p_{2}^{s-}-p_{1}^{s-}\right) & \text { for } p \in I_{1}^{s}, \\ F_{\xi \mid s}^{-1}\left(p+p_{1}^{s-}-p_{2}^{s-}\right) & \text { for } p \in I_{2}^{s} .\end{cases}
$$

Here, note that $p_{1}^{s+}-p_{1}^{s-}=p_{2}^{s+}-p_{2}^{s-}$. Then, $\left(S_{T}^{1}, Z^{\prime}\right)$ has the same distribution as $\left(S_{T}^{1}, Z\right)$, and $Z^{\prime}$ is cheaper than $Z$ because of the same argument in the proof of Theorem 1. Therefore, to obtain the cheapest payoff whose joint distribution with $S_{T}^{1}$ is the same as $\left(S_{T}^{1}, \xi\right)$, the terminal wealth should be the reverse order of $H_{T}$ under the condition that $S_{T}^{1}$ is known. Therefore, the $X$ defined by equation (6) is the cheapest payoff.

Next, let us prove the uniqueness. Suppose that $X^{\prime}$ is a random variable whose joint distribution with $S_{T}^{1}$ is the same as $\left(S_{T}^{1}, \xi\right)$ and is the reverse order of $H_{T}$ under the condition that $S_{T}^{1}$ is known. Then, $\mathbb{P}\left(B_{X^{\prime}}^{1}\right)=0$. For $s \in \mathbb{R}_{+} \backslash \mathcal{S}_{Z}$ and $\omega^{*} \in\left\{S_{T}^{1}=s\right\}$, define $U_{X^{\prime}}^{s}\left(\omega^{*}\right)$ as

$$
U_{X^{\prime}}^{s}\left(\omega^{*}\right)=\left\{\omega: S_{T}(\omega)=s, H_{T}(\omega)<H_{T}\left(\omega^{*}\right) \text {, and } X^{\prime}(\omega)<X^{\prime}\left(\omega^{*}\right)\right\},
$$

and let

$$
\begin{gathered}
V_{X^{\prime}}^{s}=\cup_{\omega^{*} \in\left\{S_{T}^{1}=s\right\}} U_{X^{\prime}}^{s}\left(\omega^{*}\right), \\
C_{X^{\prime}}=\left(\Omega \backslash B_{X^{\prime}}^{1}\right) \backslash\left(\cup_{s \in \mathcal{S}_{X^{\prime}}} V_{X^{\prime}}^{s}\right) .
\end{gathered}
$$

Then, $\mathbb{P}\left(V_{X^{\prime}}^{s} \mid S_{T}^{1}=s\right)=0$. Because it is satisfied that

$$
\mathbb{P}\left(\cup_{s \in \mathcal{S}_{X^{\prime}}} V_{X^{\prime}}^{s}\right)=\int_{\mathcal{S}_{X^{\prime}}} \mathbb{P}\left(V_{X^{\prime}}^{s} \mid S_{T}^{1}=s\right) F_{S_{T}^{1}}(d s)=0,
$$

$\mathbb{P}\left(C_{X^{\prime}}\right)=1$. For any $s \in \mathbb{R}_{+} \backslash \mathcal{S}_{Z}$ and $\omega^{*} \in C_{X^{\prime}}$,

$$
\mathbb{P}\left\{\omega: L_{T}(\omega) \leq L_{T}\left(\omega^{*}\right) \mid S_{T}^{1}=s\right\}=\mathbb{P}\left\{\omega: X^{\prime}(\omega) \leq X^{\prime}\left(\omega^{*}\right) \mid S_{T}^{1}=s\right\} .
$$

Therefore,

$$
F_{L_{T} \mid s}\left(L_{T}\left(\omega^{*}\right)\right)=F_{\xi \mid s}\left(X^{\prime}\left(\omega^{*}\right)\right) .
$$

By operating $F_{\xi \mid s}^{-1}$ to both of the sides of the equation,

$$
F_{\xi \mid s}^{-1}\left(F_{L_{T} \mid s}\left(L_{T}\left(\omega^{*}\right)\right)\right)=X^{\prime}\left(\omega^{*}\right) .
$$

Hence, $X^{\prime}=X$. 


\subsection{Derivation of the Dynamic Replicating Portfolios}

For the case of the Markovian coefficients, the concrete expressions for the cheapest dynamic portfolios can be obtained. ${ }^{2}$ Suppose that a $k$-dimensional state variable $Y_{t}$ follows SDE

$$
d Y_{t}=\mu^{Y}\left(Y_{t}\right) d t+\sigma^{Y}\left(Y_{t}\right) d W_{t}
$$

and $r_{t}, \mu_{t}$, and $\sigma_{t}$ can be described by differentiable functions of state variable $Y_{t}: r_{t}=r\left(Y_{t}\right), \mu_{t}=\mu\left(Y_{t}\right)$, and $\sigma_{t}=\sigma\left(Y_{t}\right)$. The following proposition gives the dynamic portfolios that generates the payoffs.

Proposition 2 Assume that $r, \mu$ and $\sigma$ are functions of $Y_{t}$ following SDE (15). Then, in a complete market $\mathcal{M}$, the dynamic portfolio that generates payoff $f\left(L_{T}\right)$ is given by

$$
\pi_{t}^{M}=\sigma^{\prime}(t)^{-1} \phi_{t}^{M}
$$

where

$$
\phi_{t}^{M}=X_{t} \theta_{t}+\frac{1}{H_{t}} E\left[H_{T}\left\{f^{\prime}\left(L_{T}\right)-X_{T}\right\} D_{L_{T}} \mid \mathcal{F}_{t}\right] .
$$

$D_{L_{T}}$ is given by

$$
\begin{aligned}
D_{L_{T}}= & \int_{t}^{T} \partial r\left(Y_{s}\right) A_{t, s} \sigma^{Y}\left(Y_{t}\right) d s+\int_{t}^{T} \sum_{j=1}^{n} \theta^{j}\left(Y_{s}\right) \partial \theta^{j}\left(Y_{s}\right) A_{t, s} \sigma^{Y}\left(Y_{t}\right) d s \\
& +\int_{t}^{T} \sum_{j=1}^{n} \partial \theta\left(Y_{s}\right) A_{t, s} \sigma^{Y}\left(Y_{t}\right) d W_{s}^{j}+\theta\left(Y_{t}\right)^{\prime}
\end{aligned}
$$

where $A_{t, s}$ is a $k \times k$-valued unique solution of the $S D E$

$$
d A_{t, s}=\sum_{i=1}^{n} \partial \sigma_{i}^{Y}\left(Y_{s}\right) A_{t, s} d W_{s}^{i}
$$

with an initial condition $A_{t, t}=I . \sigma_{i}^{Y}(\cdot)$ and $I$ denotes the $i$-th row of the matrix $\sigma^{Y}(\cdot)$ and the $k \times k$ identity matrix, respectively.

\footnotetext{
${ }^{2}$ The martingale method with Malliavin calculus easily gives us the dynamic portfolio that generate the payoffs. See, for example, Karatzas and Shreve (1998) for the basics of the martingale method and Nualart (2006) for the introduction to Malliavin calculus. As for the application of Malliavin calculus to the dynamic optimal portfolio and its closed form evaluation, see, for example, Ocone and Karatzas (1991) and Takahashi and Yoshida (2004).
} 
The portfolio that attains payoff $g\left(S_{T}^{1}, L_{T}\right)$ is given by

$$
\pi_{t}^{J}=\sigma^{\prime}(t)^{-1} \phi_{t}^{J}
$$

where

$$
\phi_{t}^{J}=X_{t} \theta_{t}+\frac{1}{H_{t}} E\left[H_{T} g_{1}\left(S_{T}^{1}, L_{T}\right) D_{S_{T}^{1}}+H_{T}\left\{g_{2}\left(S_{T}^{1}, L_{T}\right)-X_{T}\right\} D_{L_{T}} \mid \mathcal{F}_{t}\right] .
$$

$g_{i}(i=1,2)$ represents the partial derivative of $g$ with respect to the $i$-th argument. $D_{S_{T}^{1}}$ is given by

$$
\begin{aligned}
D_{S_{T}^{1}}= & S_{T}^{1} \int_{t}^{T}\left\{\partial \mu^{1}\left(Y_{s}\right)-\sigma^{11}\left(Y_{s}\right) \partial \sigma^{11}\left(Y_{u}\right)\right\} A_{t, s} \sigma^{Y}\left(Y_{t}\right) d s \\
& +S_{T}^{1} \int_{t}^{T} \partial \sigma^{11}\left(Y_{s}\right) A_{t, s} \sigma^{Y}\left(Y_{t}\right) d W_{s}^{1} \\
& +\left(S_{T}^{1} \sigma^{11}\left(Y_{t}\right), 0, \cdots, 0\right) .
\end{aligned}
$$

Proof. By the argument in Karatzas and Shreve (1998), $\pi_{t}$ is described as

$$
\pi_{t}=\sigma_{t}^{\prime-1}\left(X_{t} \theta_{t}+\frac{\psi_{t}}{H_{t}}\right),
$$

where $\psi_{t}$ is given by the following martingale representation

$$
M_{t}=E\left[H_{T} X_{T} \mid \mathcal{F}_{t}\right]=x+\int_{0}^{t} \psi_{u}^{\prime} d W_{u} .
$$

Applying the Clark-Ocone formula (see, for example, pp. 46 in Nualart $(2006)), \psi_{t}$ is given by

$$
\psi_{t}=E\left[\mathcal{D}_{t} \eta \mid \mathcal{F}_{t}\right]
$$

where $\eta=H_{T} X_{T}$, and $\mathcal{D}_{t}$ denotes the Malliavin derivative: $\mathcal{D}_{t} \eta=$ $\left(\mathcal{D}_{1 t} \eta, \cdots, \mathcal{D}_{n t} \eta\right)$.

To generate the marginal payoff distribution, $X_{T}=f\left(L_{T}\right)$. Then, $\mathcal{D}_{t} \eta$ can be calculated as

$$
\mathcal{D}_{t} \eta=\left(\mathcal{D}_{t} H_{T}\right) X_{T}+H_{T} f^{\prime}\left(L_{T}\right) \mathcal{D}_{t} L_{T}
$$

To generate the joint distribution with the investor's portfolio, $X_{T}=$ $g\left(S_{T}^{1}, L_{T}\right)$. Then, $\mathcal{D}_{t} \eta$ can be calculated as

$$
\mathcal{D}_{t} \eta=\left(\mathcal{D}_{t} H_{T}\right) X_{T}+H_{T} g_{1}\left(S_{T}^{1}, L_{T}\right) \mathcal{D}_{t} S_{T}^{1}+H_{T} g_{2}\left(S_{T}^{1}, L_{T}\right) \mathcal{D}_{t} L_{T},
$$


where $g_{i}(i=1,2)$ represents the partial derivative of $g$ with respect to the $i$-th argument.

$\mathcal{D}_{t} H_{T}, \mathcal{D}_{t} L_{T}$ and $\mathcal{D}_{t} S_{T}^{1}$ are calculated as

$$
\begin{gathered}
\mathcal{D}_{t} H_{T}=-H_{T} \mathcal{D}_{t} L_{T} \\
\mathcal{D}_{t} L_{T}=\quad \int_{t}^{T} \partial r\left(Y_{s}\right) \mathcal{D}_{t} Y_{s} d s+\int_{t}^{T} \sum_{j=1}^{n} \theta^{j}\left(Y_{s}\right) \partial \theta^{j}\left(Y_{s}\right) \mathcal{D}_{t} Y_{s} d s \\
+\int_{t}^{T} \sum_{j=1}^{n} \partial \theta\left(Y_{s}\right) \mathcal{D}_{t} Y_{s} d W_{s}^{j}+\theta\left(Y_{t}\right)^{\prime} \\
\mathcal{D}_{t} S_{T}^{1}=\quad S_{T}^{1} \int_{t}^{T}\left\{\partial \mu^{1}\left(Y_{s}\right)-\sigma^{11}\left(Y_{s}\right) \partial \sigma^{11}\left(Y_{u}\right)\right\} \mathcal{D}_{t} Y_{s} d s \\
+\quad S_{T}^{1} \int_{t}^{T} \partial \sigma^{11}\left(Y_{s}\right) \mathcal{D}_{t} Y_{s} d W_{s}^{1} \\
+\left(S_{T}^{1} \sigma^{11}\left(Y_{t}\right), 0, \cdots, 0\right) .
\end{gathered}
$$

In this case, $\mathcal{D}_{t} Y_{s}$ can be calculated concretely. Let $A_{t, s}$ be a $k \times k$-valued unique solution of the SDE

$$
d A_{t, s}=\sum_{i=1}^{n} \partial \sigma_{i}^{Y}\left(Y_{s}\right) A_{t, s} d W_{s}^{i}
$$

with the initial condition $A_{t, t}=I$, where $\sigma_{i}^{Y}(\cdot)$ denotes the $i$-th row of matrix $\sigma^{Y}(\cdot)$, and $I$ is the $k \times k$ identity matrix. $\mathcal{D}_{t} Y_{s}$ is represented as

$$
\mathcal{D}_{t} Y_{s}=A_{t, s} \sigma^{Y}\left(Y_{t}\right)
$$

(see, for example, pp. 126 in Nualart (2006)). Therefore, the proposition is obtained.

Proposition 1 in the main text can be directly derived from this proposition. 